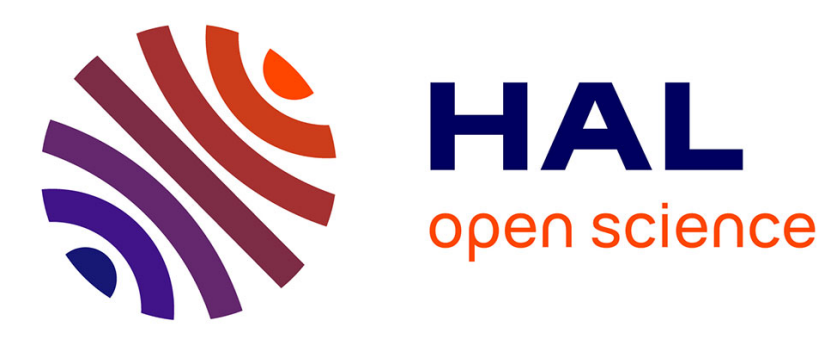

\title{
Elasto-Acoustic Coupling Between Two Circular Cylinders and Dense Fluid
}

\author{
Ilyes Mnassri, Adil El Baroudi
}

\section{To cite this version:}

Ilyes Mnassri, Adil El Baroudi. Elasto-Acoustic Coupling Between Two Circular Cylinders and Dense Fluid. International Journal of Applied Mechanics, 2017, 09 (05), pp.1750062. 10.1142/S1758825117500624 . hal-02305106

\section{HAL Id: hal-02305106 https://hal.science/hal-02305106}

Submitted on 3 Oct 2019

HAL is a multi-disciplinary open access archive for the deposit and dissemination of scientific research documents, whether they are published or not. The documents may come from teaching and research institutions in France or abroad, or from public or private research centers.
L'archive ouverte pluridisciplinaire HAL, est destinée au dépôt et à la diffusion de documents scientifiques de niveau recherche, publiés ou non, émanant des établissements d'enseignement et de recherche français ou étrangers, des laboratoires publics ou privés. 


\title{
Elasto-Acoustic Coupling Between Two Circular Cylinders and Dense Fluid
}

\author{
Ilyes Mnassri* and Adil El Baroudi ${ }^{\dagger}$ \\ Arts et Métiers ParisTech, 2 boulevard du Ronceray \\ 49035 Angers, France \\ *ilyes.mnassri@ensam.eu \\ ${ }^{\dagger}$ adil.elbaroudi@ensam.eu
}

\begin{abstract}
This paper describes a theoretical method for free vibration analysis of two elastic and isotropic cylinders filled with a dense fluid. The free vibration of two cylinders is studied on the basis of the linear three-dimensional elasticity theory. The compressible fluid is assumed to be nonviscous and isotropic which satisfy the acoustic wave equation. In this paper, the coupled dispersion equations of longitudinal, flexural and lobar modes are deduced and analytically solved. The finite element results computed by the Comsol Multiphysics software are compared with the present method for validation and an acceptable match between them was obtained. It is shown that the results from the proposed method are in good agreement with the numerical solutions. With this method, the effects of the cylinder parameters, such as the circumferential wave, the axial wavenumber, the thickness-to-radius and the length-to-radius on the coupled frequencies are investigated.
\end{abstract}

Keywords: Elasto-acoustic interaction; three-dimensional coupled vibration; thick and thin structures; frequency analysis; finite element method.

\section{Introduction}

The dynamic behavior of multilayered cylindrical structures in contact with dense fluid have been the focus of recent research, since they are extensively used as one of the basic components in many different engineering disciplines like the marine, mechanical, nuclear, aerospace, bioengineering and civil industries. Many of these studies are based on classical or thin-shell theories Leissa, 1973; Zhang, 2002. For dynamic analysis of complex structures, thin or shell theories are most appropriate, but they lose their accuracy for thick structures, making the use of three-dimensional theory of elasticity inevitable.

${ }^{\dagger}$ Corresponding author. 
The vibration analyses of multilayered cylindrical structures have been gaining an increasing attention only in the recent years. Much of the interest could be attributed to the important significance of the multilayered cylindrical structures in various practical problems contributing to noise and structural vibration. Missiles, rockets, pipes and power transmission shafts are typical cylindrical structures. When the frequency of an acting force on a cylinder coincides with it's natural frequency, the resonance phenomenon occurs and may cause failure in the hole system. Therefore, the prediction of natural frequencies is crucial and has a direct impact on the design of multilayered cylindrical structures.

The shell theory could be used for thin cylindrical structures, while thicker cylinders could only be accurately studied by using the three-dimensional elasticity theory. It should be noted that the theory of elasticity could be applied to thick or thin cylindrical structures. Gazis $1959 \mathrm{a}$ b studied the free harmonic waves in a hollow cylinder of circular cross-section, within the framework of the linear elasticity theory. He derived a characteristic equation using the Helmtholtz potentials. Armenakas [1967] derived the frequency equation for harmonic waves, with an arbitrary number of circumferential nodes, traveling in traction-free composite, circular cylindrical structures. Armenakas et al. [1969. presented the transmission of elastic energy by means of elastic waves, and formulated the eigenvalue problem for stress-free cylinders for different circumferential wavenumbers. Their analysis was based on the linear three-dimensional elasticity theory. Xuebin 2008] presented a wave propagation approach for free vibration analysis of cylindrical shell based on Flugge classical thin shell theory. A domain decomposition technique for solving vibration problems of uniform and stepped cylindrical shells with arbitrary boundary conditions was presented in $\mathrm{Qu}$ et al. 2013. Brischetto 2014. proposed a three-dimensional free vibration analysis of multilayered structures and developed an exact solution for the differential equations of equilibrium written in general orthogonal curvilinear coordinates.

Other approaches for the vibration analysis of cylindrical structures have been proposed. McNiven et al. $[1962,1966]$ used a three-mode theory to obtain a good agreement with the exact solution based on the three-dimensional theory of elasticity. El Baroudi et al. 2014] investigated the propagation of torsional, longitudinal, flexural and lobar waves in arterial wall layers numerically by using finite element method. Hamidzadeh and Jazar [2010] studied the vibrations of thick cylindrical structures. They employed the three-dimensional elastic theory and developed the governing equations of motion in terms of Helmholtz potentials. Gladwell and Vijay 1975] used a finite element approach to the three-dimensional vibration of finite length circular cylinders. Singal and Williams [1988] developed approximate solutions for natural frequencies of thick cylinders and rings, and validated their analysis by experimental investigations. Ye et al. [2014] presented a unified method based on the three-dimensional theory of elasticity developed for the free vibration analysis of thick cylindrical shells with general end conditions and resting on elastic 
foundations. In the paper [Alibeigloo and Jafarian, 2016], a free vibration analysis of carbon nanotubes reinforced composite cylindrical shell was carried out using the three-dimensional theory of elasticity.

In most of the studies mentioned above, the frequency equation of the vibration problem was obtained without taking into account the fluid's effect. The originality of this paper is to investigate the effect of a dense fluid on the modal analysis of two elastic circular cylinders (Fig. 1). This paper also discusses the influence of some model parameters such as the axial wavenumber $k_{z}$, the circumferential mode $n$, the thickness-to-radius parameter $h / a$ and the length-to-radius parameter $L / a$ on the natural frequencies. Indeed, the frequency equation was obtained for the free vibration model using the wave propagation method. The comparison of the results by the present method with those obtained by numerical finite element method has been carried out. It is shown that the present approach is simple, robust and gives accurate natural frequencies.

\section{Formulation}

\subsection{Governing equations}

As shown in Fig. 1] an isotropic circular cylinder of finite length with two layers filled with a compressible fluid in the cylindrical coordinate system $(r, \theta, z)$ is considered in the present work. The thickness of every layer is determined by radius $a, a_{1}$ and $a_{2}$ where $a$ and $a_{2}$ are the radius of the inner and outer surfaces of the cylinder, respectively. It is assumed that the cylinder is free on the outer surface. The layers are assumed to be perfectly bonded. The system displacements and stresses are defined by the cylindrical coordinates $r, \theta$ and $z$. The length of the cylinder is $L(0 \leq z \leq L)$. The values related to the inner and outer cylinders will be denoted

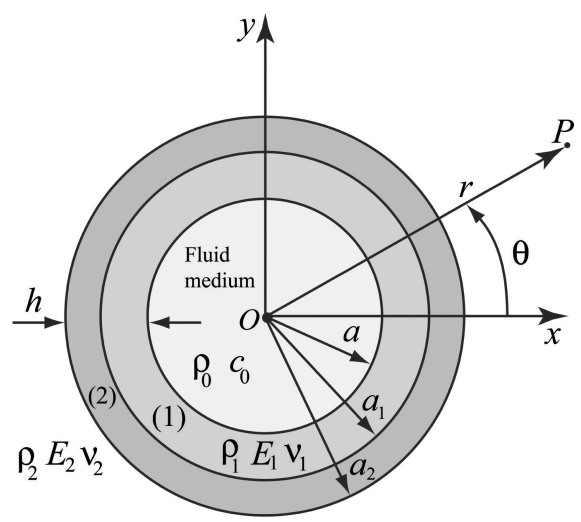

(a) The problem geometry

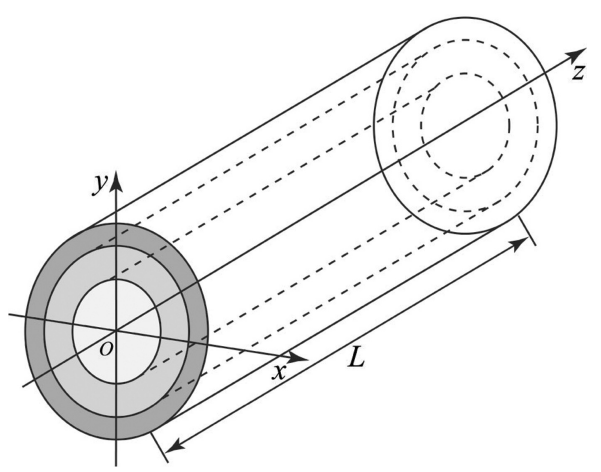

(b) Three-dimensional geometry

Fig. 1. The geometry of the cylinder with two layers filled with compressible fluid. 
by the upper indices (1) and (2), respectively. The $r, \theta$ and $z$ axes are taken in the radial, circumferential and axial directions of the cylinder, respectively.

The corresponding displacement components at any point of the cylinder in the radial, circumferential and axial directions are separately denoted by $u_{r}^{(i)}, u_{\theta}^{(i)}$ and $u_{z}^{(i)}$. According to the generalized linear Hooke's law, the elastic material under consideration is assumed to be linear, homogeneous, and isotropic for which the constitutive equation during deformation may be written as

$$
\sigma^{(i)}=\lambda_{i}\left(\nabla \cdot \mathbf{u}^{(i)}\right) \mathbf{I}+\mu_{i}\left(\nabla \mathbf{u}^{(i)}+\nabla \mathbf{u}^{(i)^{T}}\right)
$$

where $\rho_{i}$ is the mass density, $\left(\lambda_{i}, \mu_{i}\right)$ are Lamé constants, $t$ is the time and $i=1,2$ indicates the inner $(i=1)$ and the external $(i=2)$ cylinders, respectively. For the frequency analysis considered in this paper, in the absence of body forces, the displacement field is governed by the classical Navier's equation

$$
\rho_{i} \frac{\partial^{2} \mathbf{u}^{(i)}(r, \theta, z, t)}{\partial t^{2}}=\left(\lambda_{i}+2 \mu_{i}\right) \nabla \nabla \cdot \mathbf{u}^{(i)}(r, \theta, z, t)+\mu_{i} \nabla^{2} \mathbf{u}^{(i)}(r, \theta, z, t)
$$

Here, $\mathbf{u}^{(i)}=\left\{u_{r}^{(i)}, u_{\theta}^{(i)}, u_{z}^{(i)}\right\}$ is the vector displacement that can advantageously be expressed as a sum of the gradient of a scalar potential and the curl of a vector potential [Morse and Feshbach, 1946]

$$
\mathbf{u}^{(i)}=\nabla \phi^{(i)}+\nabla \times\left(\psi^{(i)} \hat{e}_{z}\right)+\nabla \times \nabla \times\left(\chi^{(i)} \hat{e}_{z}\right)
$$

where $\hat{e}_{z}$ is the unit vector along the axial coordinate directions and $\phi^{(i)}, \psi^{(i)}$ and $\chi^{(i)}$ are the Helmholtz potentials. Substituting Eq. (3) into Eq. (2) leads to the following second-order partial differential equation with constant coefficients:

$$
\nabla^{2} \phi^{(i)}=\frac{1}{c_{L i}^{2}} \frac{\partial^{2} \phi^{(i)}}{\partial t^{2}}, \quad \nabla^{2} \psi^{(i)}=\frac{1}{c_{T i}^{2}} \frac{\partial^{2} \psi^{(i)}}{\partial t^{2}}, \quad \nabla^{2} \chi^{(i)}=\frac{1}{c_{T i}^{2}} \frac{\partial^{2} \chi^{(i)}}{\partial t^{2}}
$$

where $c_{L i}=\sqrt{\left(\lambda_{i}+2 \mu_{i}\right) / \rho_{i}}$ and $c_{T i}=\sqrt{\mu_{i} / \rho_{i}}$ are the propagation velocities of dilatational and shear waves in the elastic solid, respectively. Furthermore, using Eq. (3), the relevant displacement components in cylindrical coordinates in terms of compressional and shear wave potentials may simply be written as

$$
\begin{aligned}
& u_{r}^{(i)}=\frac{\partial \phi^{(i)}}{\partial r}+\frac{1}{r} \frac{\partial \psi^{(i)}}{\partial \theta}+\frac{\partial^{2} \chi^{(i)}}{\partial r \partial z} \\
& u_{\theta}^{(i)}=\frac{1}{r} \frac{\partial \phi^{(i)}}{\partial \theta}-\frac{\partial \psi^{(i)}}{\partial r}+\frac{1}{r} \frac{\partial^{2} \chi^{(i)}}{\partial \theta \partial z} \\
& u_{z}^{(i)}=\frac{\partial \phi^{(i)}}{\partial z}+\frac{\partial^{2} \chi^{(i)}}{\partial z^{2}}-\nabla^{2} \chi^{(i)}
\end{aligned}
$$

Also, the pertinent stress components associated with the scalar potentials can be derived using the three-dimensional constitutive equation (1), which leads to the 
following expressions:

$$
\begin{aligned}
\sigma_{r r}^{(i)}=\mu_{i} & {\left[2 \frac{\partial^{2} \phi^{(i)}}{\partial r^{2}}+\frac{\lambda}{\mu} \nabla^{2} \phi^{(i)}+\frac{2}{r^{2}} \frac{\partial}{\partial \theta}\left(r \frac{\partial \psi^{(i)}}{\partial r}-\psi^{(i)}\right)+2 \frac{\partial^{3} \chi^{(i)}}{\partial r^{2} \partial z}\right] } \\
\sigma_{r \theta}^{(i)}=\mu_{i} & {\left[\frac{2}{r^{2}} \frac{\partial}{\partial \theta}\left(r \frac{\partial \phi^{(i)}}{\partial r}-\phi^{(i)}\right)+\nabla^{2} \psi^{(i)}-2 \frac{\partial^{2} \psi^{(i)}}{\partial r^{2}}-\frac{\partial^{2} \psi^{(i)}}{\partial z^{2}}\right.} \\
+ & \left.\frac{2}{r^{2}} \frac{\partial^{2}}{\partial \theta \partial z}\left(r \frac{\partial \chi^{(i)}}{\partial r}-\chi^{(i)}\right)\right] \\
\sigma_{r z}^{(i)}=\mu_{i} & {\left[2 \frac{\partial^{2} \phi^{(i)}}{\partial r \partial z}+\frac{1}{r} \frac{\partial^{2} \psi^{(i)}}{\partial \theta \partial z}+\frac{\partial}{\partial r}\left(2 \frac{\partial^{2} \chi^{(i)}}{\partial z^{2}}-\nabla^{2} \chi^{(i)}\right)\right] }
\end{aligned}
$$

Now, to yield a solution for the proposed problem, the specific boundary and continuity conditions must be satisfied at the interface of two-layer isotropic cylinder:

- at the solid-fluid interface, the continuity of the stress and velocity of the solid and fluid is given by

$$
\left.\sigma_{r r}^{(1)}\right|_{r=a}=-\left.p\right|_{r=a},\left.\quad \sigma_{r \theta}^{(1)}\right|_{r=a}=\left.\sigma_{r z}^{(1)}\right|_{r=a}=0,\left.\quad j \omega u_{r}^{(1)}\right|_{r=a}=\left.v_{r}\right|_{r=a}
$$

where $v_{r}$ is the fluid velocity components along radial direction and $p$ is the pressure.

- at the internal interface of layers $(i=1)$ and $(i=2)$, there is equality of each displacement and of stress:

$$
\begin{aligned}
& \left.\sigma_{r r}^{(1)}\right|_{r=a_{1}}=\left.\sigma_{r r}^{(2)}\right|_{r=a_{1}},\left.\quad \sigma_{r \theta}^{(1)}\right|_{r=a_{1}}=\left.\sigma_{r \theta}^{(2)}\right|_{r=a_{1}},\left.\quad \sigma_{r z}^{(1)}\right|_{r=a_{1}}=\left.\sigma_{r z}^{(2)}\right|_{r=a_{1}} \\
& \left.u_{r}^{(1)}\right|_{r=a_{1}}=\left.u_{r}^{(2)}\right|_{r=a_{1}},\left.\quad u_{\theta}^{(1)}\right|_{r=a_{1}}=\left.u_{\theta}^{(2)}\right|_{r=a_{1}},\left.\quad u_{z}^{(1)}\right|_{r=a_{1}}=\left.u_{z}^{(2)}\right|_{r=a_{1}}
\end{aligned}
$$

- the outside $(i=2)$ surface of the two cylinders are completely stress-free

$$
\left.\sigma_{r r}^{(2)}\right|_{r=a_{2}}=\left.\sigma_{r \theta}^{(2)}\right|_{r=a_{2}}=\left.\sigma_{r z}^{(2)}\right|_{r=a_{2}}=0
$$

We therefore examine the equations of motion for the fluid. Following the standard methods of theoretical acoustics, the field equations for an inviscid and ideal compressible medium that cannot support shear stresses may conveniently be expressed in terms of a scalar velocity potential as Pierce, 1991.

$$
\mathbf{v}=-\nabla \Phi, \quad p=-j \omega \rho_{0} \Phi, \quad \nabla^{2} \Phi+\frac{\omega^{2}}{c_{0}^{2}} \Phi=0
$$

where $c_{0}$ is the sound speed in the fluid medium and $\rho_{0}$ is the ambient density. In this way, inspection of the considered wave dispersion problem is reduced to the study of the eigenvalue problem formulated through Eqs. (4) and (10) and conditions (7)-(9). 


\subsection{Solution method and frequency equation}

\subsubsection{Solution method}

For the investigation of the dynamical problem of two-layer isotropic cylinder filled with a compressible fluid, various solution methods, including numerical (FEM and BEM for example) and analytical methods are used. In the present study, for the solution of the problem under consideration, we use both analytical and numerical methods. The resolution procedure starts with finding the exact solution of the problem and then the eigenvalue equation is obtained from the corresponding contact and boundary conditions. Finally, the solution of this eigenvalue equation is numerically computed using the Comsol Multiphysics software Comsol Multiphysics, 2016. In order to solve the free vibration problem of simply supported circular cylindrical isotropic structures, a time harmonic dependence $\exp (j \omega t)$ is assumed, where $j$ is the imaginary unit, $\omega$ the circular frequency and $t$ the time. The three subproblems consist of determining the scalar potentials $\phi^{(i)}(r, \theta, z), \psi^{(i)}(r, \theta, z)$ and $\chi^{(i)}(r, \theta, z)$ satisfying the Helmholtz equations (4). By going through the usual separation of variable technique, the general solution associated with an axial wavenumber $k_{z}$ and circumferential modal parameter $n$ in cylindrical coordinates can be expressed as

$$
\left\{\begin{array}{l}
\phi^{(i)} \\
\psi^{(i)} \\
\chi^{(i)}
\end{array}\right\}(r, \theta, z, t)=\left\{\begin{array}{l}
\phi^{(i)}(r) \sin (n \theta) \sin \left(k_{z} z\right) \\
\psi^{(i)}(r) \cos (n \theta) \sin \left(k_{z} z\right) \\
\chi^{(i)}(r) \sin (n \theta) \cos \left(k_{z} z\right)
\end{array}\right\} \exp (j \omega t)
$$

where $\phi^{(i)}(r), \psi^{(i)}(r)$ and $\chi^{(i)}(r)$ are defined as

$$
\begin{aligned}
& \phi^{(i)}(r)=A_{1}^{(i)} J_{n}\left(k_{\phi_{i}} r\right)+A_{2}^{(i)} Y_{n}\left(k_{\phi_{i}} r\right) \\
& \psi^{(i)}(r)=A_{3}^{(i)} J_{n}\left(k_{\psi_{i}} r\right)+A_{4}^{(i)} Y_{n}\left(k_{\psi_{i}} r\right) \\
& \chi^{(i)}(r)=A_{5}^{(i)} J_{n}\left(k_{\psi_{i}} r\right)+A_{6}^{(i)} Y_{n}\left(k_{\psi_{i}} r\right)
\end{aligned}
$$

In the above equations, $k_{\phi_{i}}$ is the modified wavenumber associated with the dilatational wave, $k_{\psi_{i}}$ is the modified wavenumber associated with the shear wave, $J_{n}$ and $Y_{n}$ are the $n$th order standard Bessel function of the first and the second kind, respectively; whereas $A_{1}^{(i)}, A_{2}^{(i)}, A_{3}^{(i)}, A_{4}^{(i)}, A_{1}^{(5)}$, and $A_{6}^{(i)}$ are unknown wave propagation coefficients whose solutions are determined below. Note that the right axial wavenumber $k_{z}$ is found by satisfying the required boundary conditions at the two ends of the hollow cylinder. The modified dilatational and shear wavenumbers are calculated using

$$
k_{\phi_{i}}=\sqrt{\frac{\omega^{2}}{c_{L i}^{2}}-k_{z}^{2}}, \quad k_{\psi_{i}}=\sqrt{\frac{\omega^{2}}{c_{T i}^{2}}-k_{z}^{2}}
$$

in which $k_{z}$ is the wavenumber in the $z$-direction. At each end of the two layered cylinder, two boundary conditions must be specified. For the simply supported 
cylinders, the following boundary conditions are imposed:

$$
\left.u_{r}^{(i)}\right|_{z=0}=\left.u_{\theta}^{(i)}\right|_{z=0}=\left.u_{r}^{(i)}\right|_{z=L}=\left.u_{\theta}^{(i)}\right|_{z=L}=0
$$

For these two boundary conditions, it can be easily shown that the axial wavenumber $k_{z}$ is a function of axial mode $m$ and cylinder length $L$ as $k_{z}=m \pi / L$. The term $k_{z} c_{T_{i}}$ defined by Eq. (12) is a frequency parameter for torsional modes when the shear wavenumber $k_{\psi_{i}}$ is taken to be equal to zero. These frequencies can be plotted as the functions of the ratio $L / a$ for different values of the longitudinal wavenumber $m$. The torsional mode is characterized by a continued rotation in a single direction. This rotation direction is continued through the length of the longitudinal wave. Therefore, the torsional wave problem does not involve the Bessel equations. Direct substitution of field expansions (11) into the field equation (5) leads to

$$
\left\{\begin{array}{l}
u_{r}^{(i)} \\
u_{\theta}^{(i)} \\
u_{z}^{(i)}
\end{array}\right\}(r, \theta, z, t)=\left\{\begin{array}{l}
u_{r}^{(i)}(r) \sin (n \theta) \sin \left(k_{z} z\right) \\
u_{\theta}^{(i)}(r) \cos (n \theta) \sin \left(k_{z} z\right) \\
u_{z}^{(i)}(r) \sin (n \theta) \cos \left(k_{z} z\right)
\end{array}\right\} \exp (j \omega t)
$$

where $u_{r}^{(i)}(r), u_{\theta}^{(i)}(r)$ and $u_{z}^{(i)}(r)$ are defined as

$$
\begin{aligned}
& u_{r}^{(i)}(r)=\phi^{(i)^{\prime}}(r)-\frac{n}{r} \psi^{(i)}(r)-k_{z} \chi^{(i)^{\prime}}(r) \\
& u_{\theta}^{(i)}(r)=\frac{n}{r} \phi^{(i)}(r)-\psi^{(i)^{\prime}}(r)-\frac{n k_{z}}{r} \chi^{(i)}(r) \\
& u_{z}^{(i)}(r)=k_{z} \phi^{(i)}(r)+k_{\psi_{i}}^{2} \chi^{(i)}(r)
\end{aligned}
$$

where the prime signifies the differentiation with respect to the argument $r$. Then, the stress $\sigma^{(i)}$ can be readily obtained using Eqs. (6) and (11)

$$
\left\{\begin{array}{c}
\sigma_{r r}^{(i)} \\
\sigma_{r \theta}^{(i)} \\
\sigma_{r z}^{(i)}
\end{array}\right\}(r, \theta, z, t)=\left\{\begin{array}{c}
\sigma_{r r}^{(i)}(r) \sin (n \theta) \sin \left(k_{z} z\right) \\
\sigma_{r \theta}^{(i)}(r) \cos (n \theta) \sin \left(k_{z} z\right) \\
\sigma_{r z}^{(i)}(r) \sin (n \theta) \cos \left(k_{z} z\right)
\end{array}\right\} \exp (j \omega t)
$$

where $\sigma_{r r}^{(i)}(r), \sigma_{r \theta}^{(i)}(r)$ and $\sigma_{r z}^{(i)}(r)$ are defined as

$$
\begin{aligned}
\sigma_{r r}^{(i)}(r)= & \mu_{i}\left\{2 \phi^{(i)^{\prime \prime}}(r)-\frac{\lambda_{i}}{\mu_{i}} \frac{\omega^{2}}{c_{L i}^{2}} \phi^{(i)}(r)-\frac{2 n}{r^{2}}\left[r \psi^{(i)^{\prime}}(r)-\psi^{(i)}(r)\right]-2 k_{z} \chi^{(i)^{\prime \prime}}(r)\right\} \\
\sigma_{r \theta}^{(i)}(r)= & \mu_{i}\left\{\frac{2 n}{r^{2}}\left[r \phi^{(i)^{\prime}}(r)-\phi^{(i)}(r)\right]-2 \psi^{(i)^{\prime \prime}}(r)-k_{\psi_{i}}^{2} \psi^{(i)}(r)\right. \\
& \left.-\frac{2 n k_{z}}{r^{2}}\left[r \chi^{(i)^{\prime}}(r)-\chi^{(i)}(r)\right]\right\} \\
\sigma_{r z}^{(i)}(r)= & \mu_{i}\left[2 k_{z} \phi^{(i)^{\prime}}(r)-\frac{n k_{z}}{r} \psi^{(i)}(r)+\left(k_{\psi_{i}}^{2}-k_{z}^{2}\right) \chi^{(i)^{\prime}}(r)\right]
\end{aligned}
$$


Similarly, the solution for Eq. (11) which belongs to the longitudinal and shear waves inside the compressible fluid is represented in cylindrical coordinates by

$$
\Phi(r, \theta, z, t)=A J_{n}\left(k_{\Phi} r\right) \sin (n \theta) \sin \left(k_{z} z\right) \exp (j \omega t), \quad k_{\Phi}=\sqrt{\frac{\omega^{2}}{c_{0}^{2}}-k_{z}^{2}}
$$

where $J_{n}\left(k_{\Phi} r\right)$ is the Bessel function of the first kind and $A$ is the unknown modal coefficient. Note that $\phi^{(i)}$ (or $\left.\psi^{(i)}, \chi^{(i)}, \Phi\right)$ has components that are symmetric or antisymmetric in $\theta$ and $z$. Following standard practice, the solutions with symmetric (antisymmetric) axial $\phi^{(i)}$ are called the antisymmetric (symmetric) axial modes, respectively, with $k_{z}^{a}$ and $k_{z}^{s}$ denoting the corresponding eigenvalues. Thus, we have $k_{z}^{a}=\left(m-\frac{1}{2}\right) \pi / L$ and $k_{z}^{s}=m \pi / L$. However, the azimuthal modes corresponding to $\cos (n \theta)$ and $\sin (n \theta)$ are really the same, due to periodicity in the azimuthal direction, i.e., there is no distinction in the values of $n$ for the two families.

\subsubsection{Frequency equation}

Now, we shall derive the frequency equation for the isotropic circular cylinder. Combining the continuity and boundary conditions (7)-(9) with Eqs. (13)-(15) yields for each vector mode number $(n, m)$ to the following linear system:

$\mathbf{N Y}=\mathbf{0}$

$$
\mathbf{Y}=\left\{A_{1}^{(1)}, A_{2}^{(1)}, A_{3}^{(1)}, A_{4}^{(1)}, A_{5}^{(1)}, A_{6}^{(1)}, A_{1}^{(2)}, A_{2}^{(2)}, A_{3}^{(2)}, A_{4}^{(2)}, A_{5}^{(2)}, A_{6}^{(2)}, A\right\}^{T}
$$

The matrix $\mathbf{N}$ is defined as follows:

$$
\mathbf{N}=\left[\begin{array}{ccccccccccccc}
N_{11}^{(1)} & N_{12}^{(1)} & N_{13}^{(1)} & N_{14}^{(1)} & N_{15}^{(1)} & N_{16}^{(1)} & N_{11}^{(2)} & N_{12}^{(2)} & N_{13}^{(2)} & N_{14}^{(2)} & N_{15}^{(2)} & N_{16}^{(2)} & N_{1} \\
N_{21}^{(1)} & N_{22}^{(1)} & N_{23}^{(1)} & N_{24}^{(1)} & N_{25}^{(1)} & N_{26}^{(1)} & N_{21}^{(2)} & N_{22}^{(2)} & N_{23}^{(2)} & N_{24}^{(2)} & N_{25}^{(2)} & N_{26}^{(2)} & 0 \\
N_{31}^{(1)} & N_{32}^{(1)} & N_{33}^{(1)} & N_{34}^{(1)} & N_{35}^{(1)} & N_{36}^{(1)} & N_{31}^{(2)} & N_{32}^{(2)} & N_{33}^{(2)} & N_{34}^{(2)} & N_{35}^{(2)} & N_{36}^{(2)} & 0 \\
N_{41}^{(1)} & N_{42}^{(1)} & N_{43}^{(1)} & N_{44}^{(1)} & N_{45}^{(1)} & N_{46}^{(1)} & N_{41}^{(2)} & N_{42}^{(2)} & N_{43}^{(2)} & N_{44}^{(2)} & N_{45}^{(2)} & N_{46}^{(2)} & N_{4} \\
N_{51}^{(1)} & N_{52}^{(1)} & N_{53}^{(1)} & N_{54}^{(1)} & N_{55}^{(1)} & N_{56}^{(1)} & N_{51}^{(2)} & N_{52}^{(2)} & N_{53}^{(2)} & N_{54}^{(2)} & N_{55}^{(2)} & N_{56}^{(2)} & 0 \\
N_{61}^{(1)} & N_{62}^{(1)} & N_{63}^{(1)} & N_{64}^{(1)} & N_{65}^{(1)} & N_{66}^{(1)} & N_{61}^{(2)} & N_{62}^{(2)} & N_{63}^{(2)} & N_{64}^{(2)} & N_{65}^{(2)} & N_{66}^{(2)} & 0 \\
N_{71}^{(1)} & N_{72}^{(1)} & N_{73}^{(1)} & N_{74}^{(1)} & N_{75}^{(1)} & N_{76}^{(1)} & N_{71}^{(2)} & N_{72}^{(2)} & N_{73}^{(2)} & N_{74}^{(2)} & N_{75}^{(2)} & N_{76}^{(2)} & 0 \\
N_{81}^{(1)} & N_{82}^{(1)} & N_{83}^{(1)} & N_{84}^{(1)} & N_{85}^{(1)} & N_{86}^{(1)} & N_{81}^{(2)} & N_{82}^{(2)} & N_{83}^{(2)} & N_{84}^{(2)} & N_{85}^{(2)} & N_{86}^{(2)} & 0 \\
N_{91}^{(1)} & N_{92}^{(1)} & N_{93}^{(1)} & N_{94}^{(1)} & N_{95}^{(1)} & N_{96}^{(1)} & N_{91}^{(2)} & N_{92}^{(2)} & N_{93}^{(2)} & N_{94}^{(2)} & N_{95}^{(2)} & N_{96}^{(2)} & 0 \\
N_{101}^{(1)} & N_{102}^{(1)} & N_{103}^{(1)} & N_{104}^{(1)} & N_{105}^{(1)} & N_{106}^{(1)} & N_{101}^{(2)} & N_{102}^{(2)} & N_{103}^{(2)} & N_{104}^{(2)} & N_{105}^{(2)} & N_{106}^{(2)} & 0 \\
0 & 0 & 0 & 0 & 0 & 0 & N_{111}^{(2)} & N_{112}^{(2)} & N_{113}^{(2)} & N_{114}^{(2)} & N_{115}^{(2)} & N_{116}^{(2)} & 0 \\
0 & 0 & 0 & 0 & 0 & 0 & N_{121}^{(2)} & N_{122}^{(2)} & N_{123}^{(2)} & N_{124}^{(2)} & N_{125}^{(2)} & N_{126}^{(2)} & 0 \\
0 & 0 & 0 & 0 & 0 & 0 & N_{131}^{(2)} & N_{132}^{(2)} & N_{133}^{(2)} & N_{134}^{(2)} & N_{135}^{(2)} & N_{136}^{(2)} & 0
\end{array}\right]
$$


and whose elements are given in the Appendix. For nontrivial solution, the determinant of the coefficient matrix in Eq. (16) must be zero:

$$
\operatorname{det}(\mathbf{N})=0
$$

For given material and geometric properties, the expansion of Eq. (17) will give the frequency equation for a particular value of $n$. For any value of $k_{z}$, the frequency equation will yield an infinite number of values of $\omega$, each corresponding to a different mode of wave propagation. Note that the solutions for $n=0$ correspond to the longitudinal modes, together with the torsional modes, for $n=1$ to the flexural modes and for $n>1$ to the lobar modes.

\subsubsection{Longitudinal vibration}

The longitudinal mode refers to the absence of torsional displacement $u_{\theta}=0$. In this case, the displacement field is independent of the $\theta$-coordinate and is of the form $\left(u_{r}^{(i)}, 0, u_{z}^{(i)}\right)$. This mode of wave propagation corresponds to $n=0$ in Eq. (17) and results in $\psi^{(i)}=0$ [Morse and Feshbach, 1946]. Therefore, the boundary condition Eqs. (7)-(9) become

$$
\begin{aligned}
& \left.\sigma_{r r}^{(1)}\right|_{r=a}=-\left.p\right|_{r=a},\left.\quad \sigma_{r z}^{(1)}\right|_{r=a}=0,\left.\quad j \omega u_{r}^{(1)}\right|_{r=a}=\left.v_{r}\right|_{r=a} \\
& \left.\sigma_{r r}^{(1)}\right|_{r=a_{1}}=\left.\sigma_{r r}^{(2)}\right|_{r=a_{1}},\left.\quad \sigma_{r z}^{(1)}\right|_{r=a_{1}}=\left.\sigma_{r z}^{(2)}\right|_{r=a_{1}} \\
& \left.u_{r}^{(1)}\right|_{r=a_{1}}=\left.u_{r}^{(2)}\right|_{r=a_{1}},\left.\quad u_{z}^{(1)}\right|_{r=a_{1}}=\left.u_{z}^{(2)}\right|_{r=a_{1}} \\
& \left.\sigma_{r r}^{(2)}\right|_{r=a_{2}}=\left.\sigma_{r z}^{(2)}\right|_{r=a_{2}}=0
\end{aligned}
$$

Then, Eq. (16) becomes

$$
\mathbf{N}_{L} \mathbf{Y}_{L}=\mathbf{0}, \quad \mathbf{Y}_{L}=\left\{A_{1}^{(1)}, A_{2}^{(1)}, A_{5}^{(1)}, A_{6}^{(1)}, A_{1}^{(2)}, A_{2}^{(2)}, A_{5}^{(2)}, A_{6}^{(2)}, A\right\}^{T}
$$

where

$$
\mathbf{N}_{L}=\left[\begin{array}{ccccccccc}
N_{11}^{(1)} & N_{12}^{(1)} & N_{15}^{(1)} & N_{16}^{(1)} & N_{11}^{(2)} & N_{12}^{(2)} & N_{15}^{(2)} & N_{16}^{(2)} & N_{1} \\
N_{31}^{(1)} & N_{32}^{(1)} & N_{35}^{(1)} & N_{36}^{(1)} & N_{31}^{(2)} & N_{32}^{(2)} & N_{35}^{(2)} & N_{36}^{(2)} & 0 \\
N_{41}^{(1)} & N_{42}^{(1)} & N_{45}^{(1)} & N_{46}^{(1)} & N_{41}^{(2)} & N_{42}^{(2)} & N_{45}^{(2)} & N_{46}^{(2)} & N_{4} \\
N_{51}^{(1)} & N_{52}^{(1)} & N_{55}^{(1)} & N_{56}^{(1)} & N_{51}^{(2)} & N_{52}^{(2)} & N_{55}^{(2)} & N_{56}^{(2)} & 0 \\
N_{71}^{(1)} & N_{72}^{(1)} & N_{75}^{(1)} & N_{76}^{(1)} & N_{71}^{(2)} & N_{72}^{(2)} & N_{75}^{(2)} & N_{76}^{(2)} & 0 \\
N_{81}^{(1)} & N_{82}^{(1)} & N_{85}^{(1)} & N_{86}^{(1)} & N_{81}^{(2)} & N_{82}^{(2)} & N_{85}^{(2)} & N_{86}^{(2)} & 0 \\
N_{101}^{(1)} & N_{102}^{(1)} & N_{105}^{(1)} & N_{106}^{(1)} & N_{101}^{(2)} & N_{102}^{(2)} & N_{105}^{(2)} & N_{106}^{(2)} & 0 \\
0 & 0 & 0 & 0 & N_{111}^{(2)} & N_{112}^{(2)} & N_{115}^{(2)} & N_{116}^{(2)} & 0 \\
0 & 0 & 0 & 0 & N_{131}^{(2)} & N_{132}^{(2)} & N_{135}^{(2)} & N_{136}^{(2)} & 0
\end{array}\right]
$$

Solving $\operatorname{det}\left(\mathbf{N}_{L}\right)=0$ gives the frequency equation for longitudinal modes. 


\subsubsection{Finite element modeling}

In this section, the commercially available FEA package COMSOL Multiphysics was used to develop the finite element model of an elasto-acoustic coupling between two circular cylinders and dense fluid. This modeling procedure requires two modules: one for simulating the elastic tube and the other for the dense fluid. Each module provides a wide range of equations, which was needed in specifying subdomains and boundaries. The theories and equations behind this model are based on the governing equations in Sec. 2 The cylinders were considered to be a threedimensional solid, stress-strain problem. The elastic cylinders and fluid were simulated using Lagrange-Quadratic element and Lagrange- $P_{2} P_{1}$ element, respectively. The cylinders and fluid elements at the interface shared the same nodes and had extremely fine meshes to capture the details during the coupled vibrations. The applied boundary conditions were as follows. The cylinders were simply supported at two ends $\left(u_{x}=u_{y}=0\right)$. The fluid pressure nodes at the two ends were constrained $(p=0)$. Moreover, for simulation of the coupled system, two modules (Fluid Flow and Structural Mechanics) were used. Two elastic cylinders were used to envelope the fluid domain. On the outer cylindrical perimeter of the solid domain, a free constraint condition was used. The coupling of elastic cylinders' vibration with the dense fluid inside the cylinders was performed using both Fluid Flow and Structural Mechanic modules. For this purpose, some variables were set to make the connection between these two modules. At fluid-structure interface, kinematic and dynamic continuity have to be ensured. The complete coupled problem has to fulfil the condition that the location of the fluid-structure interface coincides for both the fields. Thus, the fluid-structure interaction boundary condition concerning the fluid is of an inhomogeneous Dirichlet type (kinematic condition) which represents the mass conservation across the interface, whereas the fluid-structure boundary condition for the solid is given by an inhomogeneous Neumann condition (dynamic condition) which describes the equivalence of fluid stresses and solid stresses in normal direction. For the modeling of the coupled system, eigenfrequency solver was selected to solve the model to give the first eight eigenvalues.

\section{Results and Validation}

The frequency equation (17) gives the frequency equations of the lobar, flexural, longitudinal and torsional modes in terms of its elastic constants. We consider twolayer circular cylinder filled with a compressible fluid as shown in Fig. 1 and assume that the radius of the internal circle of the inner cylinder is $a$ and the thickness of the middle and outer cylinders is $h_{1}=a_{1}-a$ and $h_{2}=a_{2}-a_{1}$, respectively. The resulting frequency equation given in Eq. (17) is transcendental in nature with respect to the frequency $\omega$ and wavenumber $k_{z}$. To obtain the roots of the frequency equation, the Mathematica software was used [Mathematica, 2015]. To validate the analytical results, the natural frequencies and mode shapes are also computed using Comsol 
Multiphysics FEM Simulation Software [Comsol Multiphysics, 2008]. To simplify the presentation of results and comparison with other established results for specific cases, natural frequencies are normalized and introduced as the nondimensional frequency, which is defined by $\Omega=\omega h /\left(\pi c_{T i}\right)$, where $h=h_{2}+h_{1}$. In order to compare the results, we maintain the same frequency factor as the one defined by Armenakas et al. [1969] and Hamidzadeh et al. [2010] in the case of uncoupled model (cylinders in the vacuum). The focus of this paper is to validate the accuracy of the method for calculating the coupled natural frequency of two circular cylinders filled with a compressible fluid.

The same geometric and material properties used for the finite circular cylinders in Hamidzadeh et al. [2010] were taken to construct this numerical example. The following values of the isotropic layer (middle layer $i=2$ ) were assumed: $\rho_{i}=7747.6\left(\mathrm{~kg} / \mathrm{m}^{3}\right)$ (density), $\nu_{i}=0.32$ (Poisson ratio) and $E_{i}=2.1 \cdot 10^{11}(\mathrm{~Pa})$ (Young's modulus). The two circular cylinders are simply supported at both ends and subjected to a compressible fluid with sound speed $c_{0}=1500(\mathrm{~m} / \mathrm{s})$ and mass density $\rho_{0}=1000\left(\mathrm{~kg} / \mathrm{m}^{3}\right)$. Uncoupled and coupled natural frequencies are obtained analytically by Mathematica [2010] and numerically by Comsol Mathematics [2008]. Table 1shows the comparison of the first eight uncoupled frequencies of the two thick cylinders by Armenakas et al. [1969] and in Hamidzadeh et al. [2010], FEM, and the present method. The uncoupled frequencies predicted by the present method are identical to those calculated by FEM and by Armenakas et al. [1969] and in Hamidzadeh et al. [2010], indicating the validity of our model. The comparisons show that the present method is correct with good accuracy. For an uncoupled shell's example, exact and approximate results for a cylindrical shell, simply supported at both ends, have been reported by Oliazadeh et al. 2013]. This shell has the following properties: $E=68.2(\mathrm{GPa}), \nu=1 / 3, \rho=2700\left(\mathrm{~kg} / \mathrm{m}^{3}\right), h / a=1 / 20$ and $L / a=3$. Table 2 shows a comparison of exact results obtained by using the present study with those of Oliazadeh et al. 2013] and calculated using FEM (Comsol Multiphysics). It can be seen generally that exact results obtained using the present study agree with the exact results using different shell theories (see Oliazadeh et al. 2013 ). In the case of two cylinders with a compressible fluid (Table 3), a very good

Table 1. Comparison of the first eight $\Omega_{n, m}=h \omega_{n, m} /\left(\pi c_{T i}\right)$ in the case of uncoupled vibration for $h / a=0.66$ and $L / a=1.33$.

\begin{tabular}{lccccl}
\hline$(n, m)$ & Present & FEM & Hamidzadeh et al. & Armenakas et al. & Mode shape \\
\hline$(1,1)$ & 0.361 & 0.361 & 0.359 & 0.359 & Flexural \\
$(0,1)$ & 0.371 & 0.371 & 0.369 & 0.369 & Longitudinal \\
$(2,1)$ & 0.375 & 0.375 & 0.373 & 0.373 & Lobar \\
$(3,1)$ & 0.444 & 0.444 & 0.441 & 0.441 & Lobar \\
$(0,1)$ & 0.499 & 0.499 & & & Torsional \\
$(1,1)$ & 0.536 & 0.536 & 0.536 & 0.536 & Flexural \\
$(4,1)$ & 0.553 & 0.553 & 0.550 & 0.550 & Lobar \\
$(2,1)$ & 0.614 & 0.614 & 0.614 & 0.614 & Lobar \\
\hline
\end{tabular}


Table 2. Comparison of the first eight $\Omega_{n, m}^{*}=\omega a \sqrt{\frac{\left(1-\nu^{2}\right) \rho}{E}}$ in the case of uncoupled vibration of shell for $h / a=1 / 20, L / a=3$ and $\nu=1 / 3$.

\begin{tabular}{lcccccc}
\hline$(n, m)$ & $\begin{array}{c}\text { Present } \\
\text { (Exact) }\end{array}$ & $\begin{array}{c}\text { FEM } \\
\text { (Comsol Multiphysics) }\end{array}$ & $\begin{array}{c}\text { Soedel } \\
\text { (Exact) }\end{array}$ & $\begin{array}{c}\text { Flugge } \\
\text { (Exact) }\end{array}$ & $\begin{array}{c}\text { Morley-Koiter } \\
\text { (Exact) }\end{array}$ & $\begin{array}{c}\text { Donnell } \\
\text { (Exact) }\end{array}$ \\
\hline$(3,1)$ & 0.1528 & 0.1528 & 0.1520 & 0.1681 & 0.1563 & 0.1563 \\
$(2,1)$ & 0.1882 & 0.1882 & 0.1863 & 0.1900 & 0.1870 & 0.1858 \\
$(3,2)$ & 0.3364 & 0.3364 & 0.3382 & 0.3453 & 0.3377 & 0.3381 \\
$(1,1)$ & 0.3690 & 0.3690 & 0.3660 & 0.3649 & 0.3653 & 0.3674 \\
$(2,2)$ & 0.4640 & 0.4641 & 0.4647 & 0.4672 & 0.4644 & 0.4642 \\
$(3,3)$ & 0.5316 & 0.5318 & 0.5370 & 0.5425 & 0.5361 & 0.5359 \\
$(2,3)$ & 0.6618 & 0.6620 & 0.6695 & 0.6718 & 0.6688 & 0.6680 \\
$(1,2)$ & 0.6972 & 0.6974 & 0.7044 & 0.7050 & 0.7043 & 0.7039 \\
$(1,3)$ & 0.8308 & 0.8310 & 0.8473 & 0.8479 & 0.8467 & 0.8455 \\
\hline
\end{tabular}

Table 3. Comparison of the first eight $\Omega_{n, m}=h \omega_{n, m} /\left(\pi c_{T i}\right)$ in the case of coupled vibration for $h / a=0.66$ and $L / a=1.33$.

\begin{tabular}{lccll}
\hline$(n, m)$ & Present & FEM & Mode shape & Order \\
\hline$(0,1)$ & 0.2225 & 0.2225 & Longitudinal & First \\
$(1,1)$ & 0.2776 & 0.2776 & Flexural & First \\
$(2,1)$ & 0.3422 & 0.3422 & Lobar & First \\
$(0,1)$ & 0.3655 & 0.3655 & Longitudinal & Second \\
$(1,1)$ & 0.3678 & 0.3678 & Flexural & Second \\
$(2,1)$ & 0.4016 & 0.4016 & Lobar & Second \\
$(3,1)$ & 0.4194 & 0.4194 & Lobar & First \\
$(0,1)$ & 0.4407 & 0.4407 & Longitudinal & Third \\
\hline
\end{tabular}

agreement was observed between the results of the present method and those of FEM throughout the very low relative difference ((FEM-Present)/Present) which is actually $\leq 0.01 \%$. Figures 25 illustrate the first three coupled modal shapes. The modal shape can be regarded as the mode $(n, m)$, where $n$ is the modal number in the circumferential direction and $m$ is the modal number in the axial direction. The modal shapes in Figs. 2 and 4 are not in order with the parameters $n$ and $m$ in the case of thin cylinders (short or long). The frequency of mode $(5,1)$ is lower than that of mode $(4,1)$, for example. This feature of thin cylinders' vibration is different from that of the beam vibration in which the order increases with the modal parameter. Therefore, in the vibration of thin cylinders, one should be careful to find the right mode of the vibration. On the other hand, in Figs. 3 and 5, the modal shapes are in order with the parameters $n$ and $m$ in the case of thick cylinders (short or long). The frequency of mode $(2,1)$ is lower than that of mode $(1,1)$, for example.

For the free vibration analysis of short (or long) and thick (or thin) two elastic and isotropic cylinders subjected to compressible fluid, the effects of cylinder parameters on the coupled natural frequencies are presented in the present method. In these studies, investigations were carried out to study the effects of circumferential mode $n$, axial wavenumber $k_{z}$, thickness-to-radius parameter $h / a$ and length-toradius parameter $L / a$ on the coupled frequencies. 


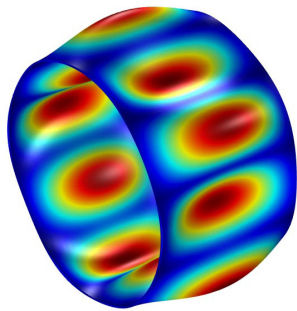

(a) $\Omega_{5,1}=0.00176$

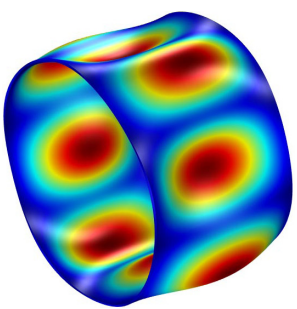

(b) $\Omega_{4,1}=0.00187$

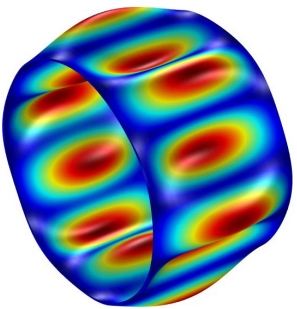

(c) $\Omega_{6,1}=0.00200$

Fig. 2. First three coupled $\Omega_{n, m}$ and associated mode shapes $(h / a=0.02$ and $L / a=1.33)$.

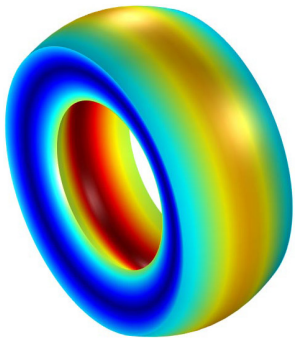

(a) $\Omega_{0,1}=0.233$

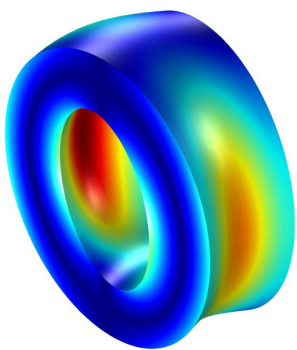

(b) $\Omega_{1,1}=0.292$

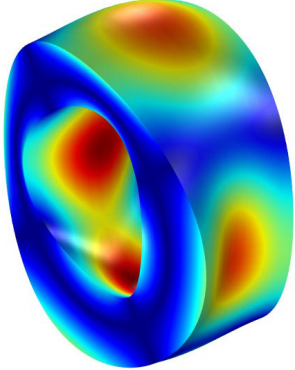

(c) $\Omega_{2,1}=0.363$

Fig. 3. First three coupled $\Omega_{n, m}$ and associated mode shapes $(h / a=0.7$ and $L / a=1.33)$.

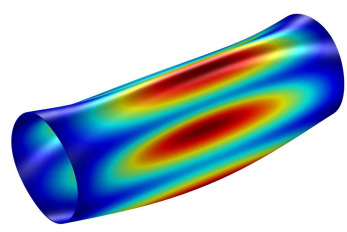

(a) $\Omega_{3,1}=0.000327$

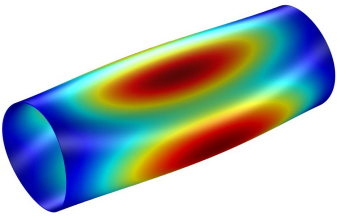

(b) $\Omega_{2,1}=0.000331$

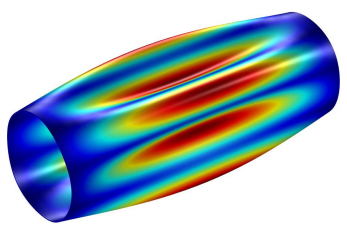

(c) $\Omega_{4,1}=0.000593$

Fig. 4. First three coupled $\Omega_{n, m}$ and associated mode shapes $(h / a=0.02$ and $L / a=6)$.

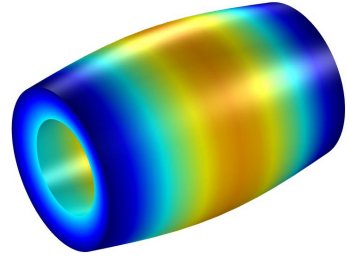

(a) $\Omega_{0,1}=0.0515$

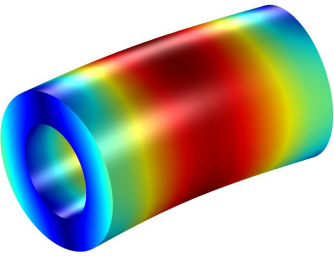

(b) $\Omega_{1,1}=0.0628$

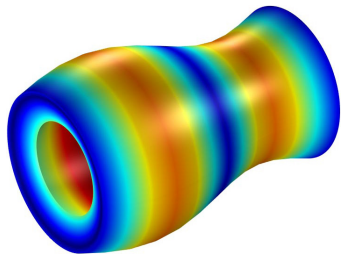

(c) $\Omega_{0,2}=0.1029$

Fig. 5. First three coupled $\Omega_{n, m}$ and associated mode shapes $(h / a=0.7$ and $L / a=6)$. 


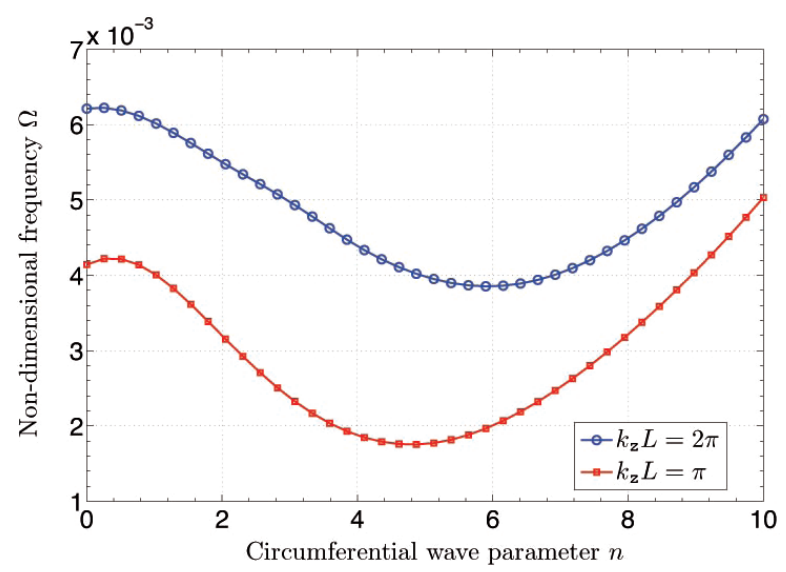

Fig. 6. Variation of coupled frequency parameter $\Omega$ versus $n$ for $h / a=0.02$ and $L / a=1.33$.

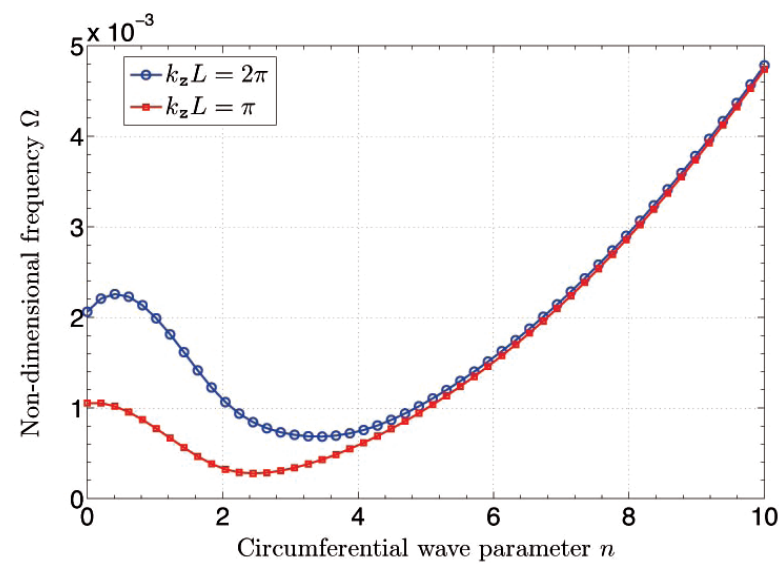

Fig. 7. Variation of coupled frequency parameter $\Omega$ versus $n$ for $h / a=0.02$ and $L / a=6$.

We begin by examining how the coupled frequencies for the given $h / a$ and $L / a$ ratios vary with the axial mode $m$ and circumferential mode $n$. Figure 6 exhibits that for $h / a=0.02$ (case of thin structures), with $L / a=1.33$ and $L / a=6$, the frequencies first decrease and then increase as the circumferential mode $n$ increases. This behavior is also found for short thick structures (Fig. 8). Figure7 7 indicates that both the $m$ frequency curves converge when $n$ is large which means that for large circumferential mode $n$, the frequencies are only determined by the $n$ and regardless of the axial mode $m$. This behavior is also found for long thick structures (Fig. (9). For a given $n$, the frequency increases as $m$ increases. Therefore, the fundamental frequency is always with the $m=1$ curve. In the following, we study the frequency curves of $m=1\left(k_{z} L=\pi\right)$ with the change of the other cylinder parameters. 


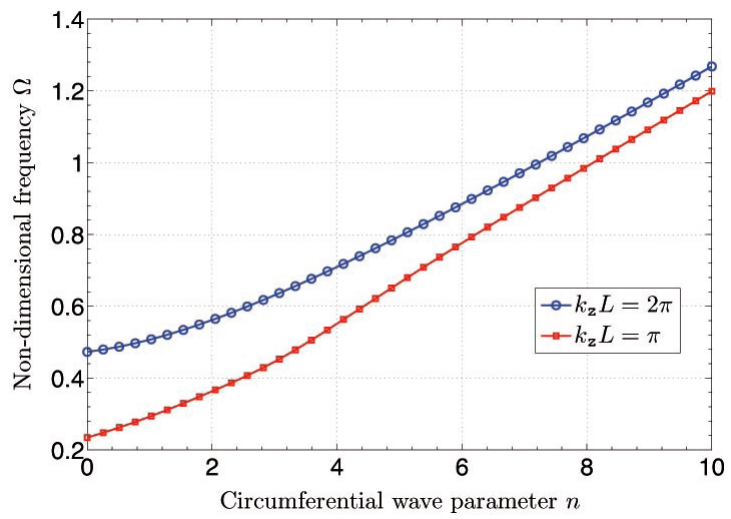

Fig. 8. Variation of coupled frequency parameter $\Omega$ versus $n$ for $h / a=0.7$ and $L / a=1.33$.

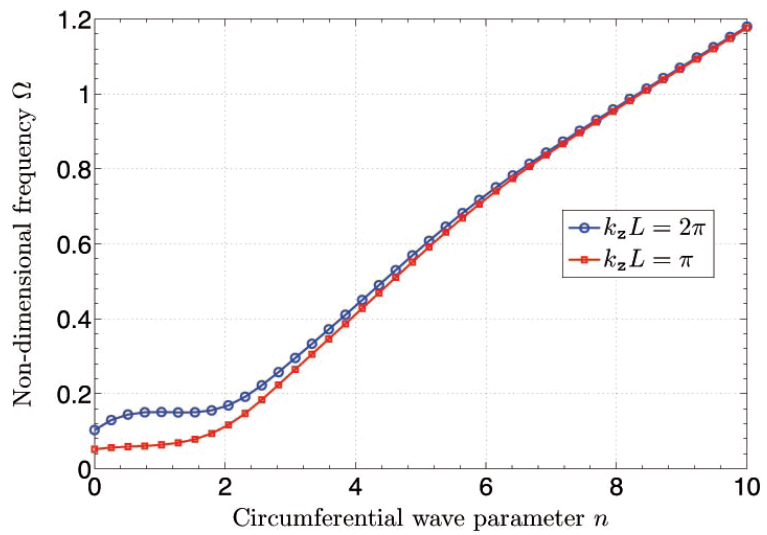

Fig. 9. Variation of coupled frequency parameter $\Omega$ versus $n$ for $h / a=0.7$ and $L / a=6$.

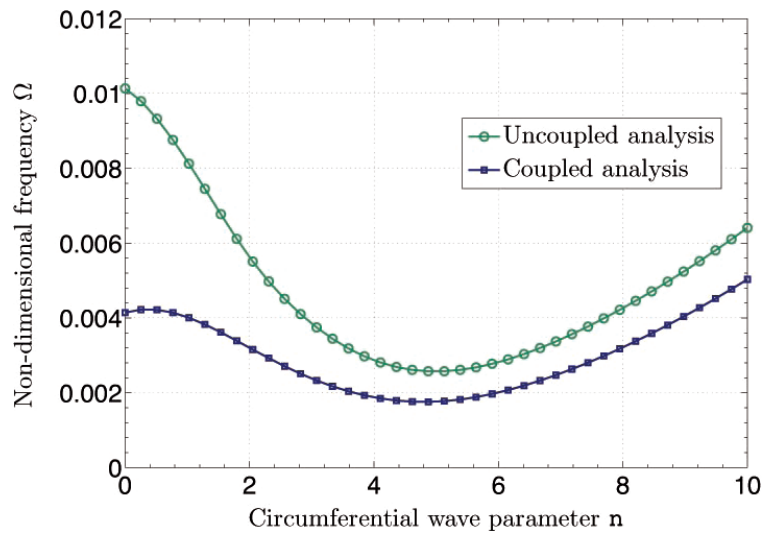

Fig. 10. Variation of frequency parameter $\Omega$ versus $n$ for $h / a=0.02$ and $L / a=1.33$. 


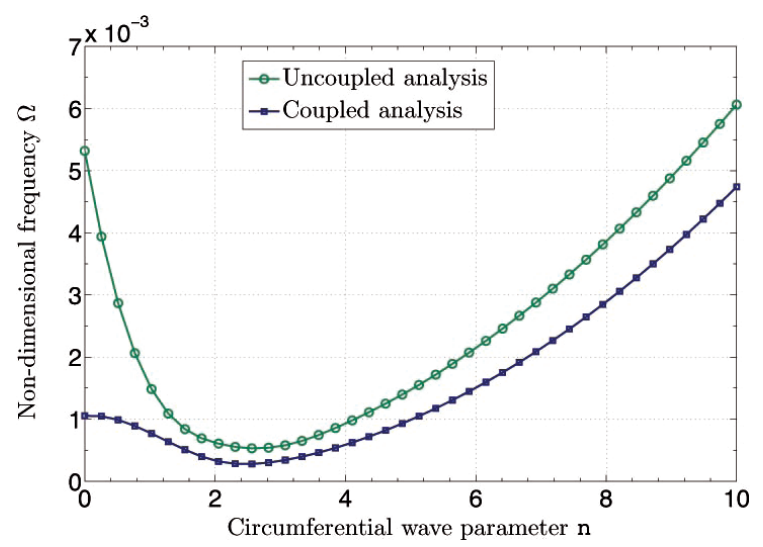

Fig. 11. Variation of frequency parameter $\Omega$ versus $n$ for $h / a=0.02$ and $L / a=6$.

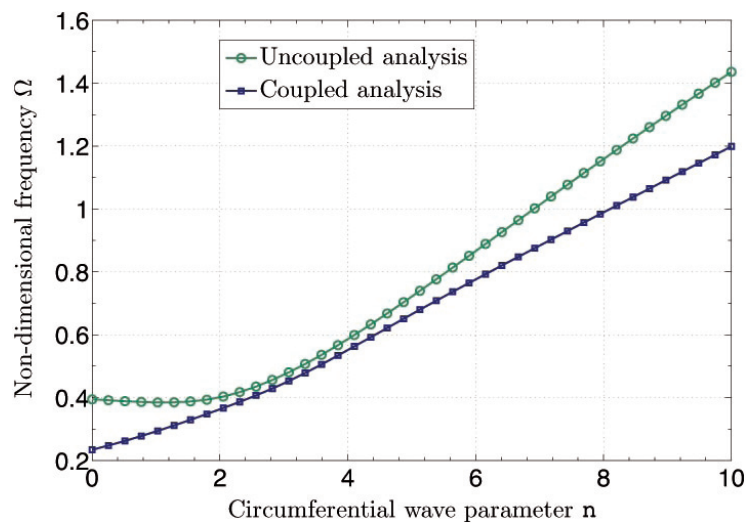

Fig. 12. Variation of frequency parameter $\Omega$ versus $n$ for $h / a=0.7$ and $L / a=1.33$.

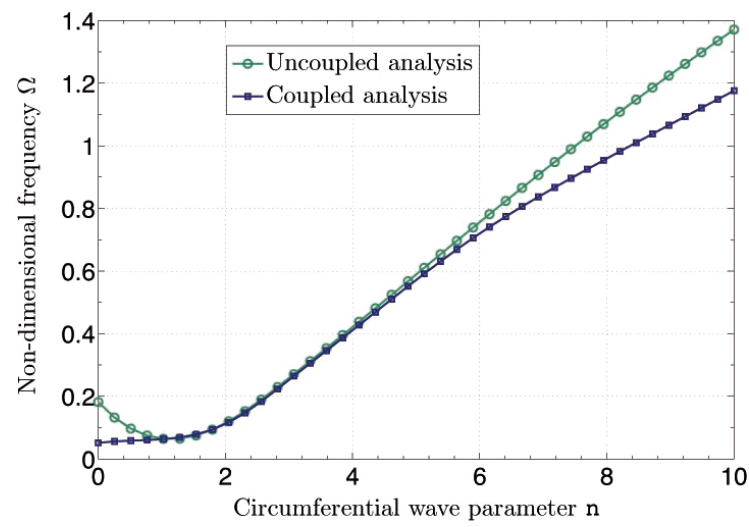

Fig. 13. Variation of frequency parameter $\Omega$ versus $n$ for $h / a=0.7$ and $L / a=6$. 


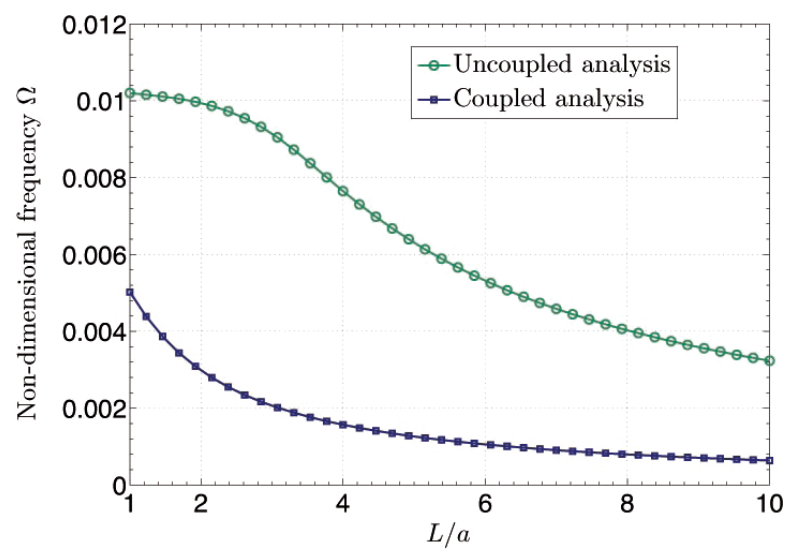

Fig. 14. Frequency curves for longitudinal modes $(n=0)$, as $\Omega$ versus $L / a$ with $h / a=0.02$.

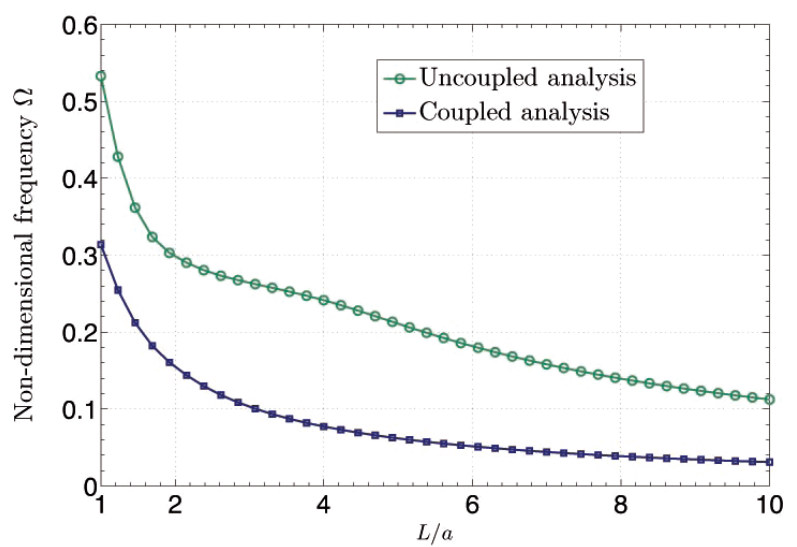

Fig. 15. Frequency curves for longitudinal modes $(n=0)$, as $\Omega$ versus $L / a$ with $h / a=0.7$.

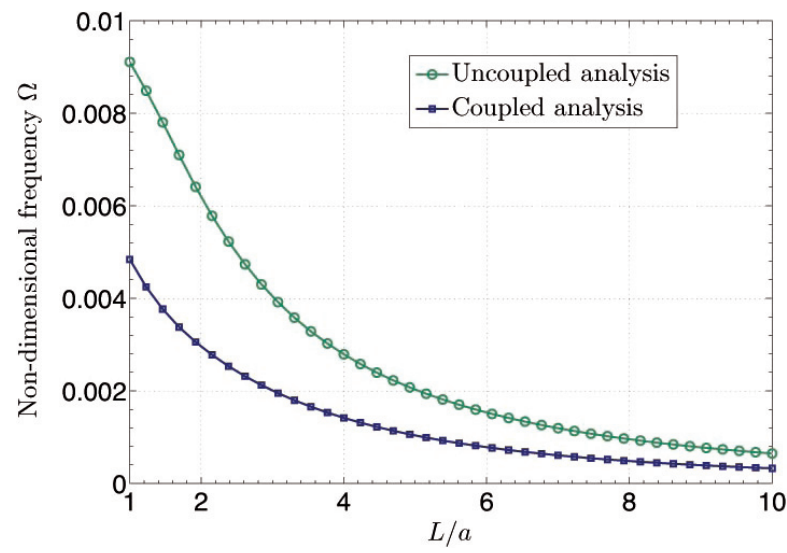

Fig. 16. Frequency curves for flexural modes $(n=1)$, as $\Omega$ versus $L / a$ with $h / a=0.02$. 


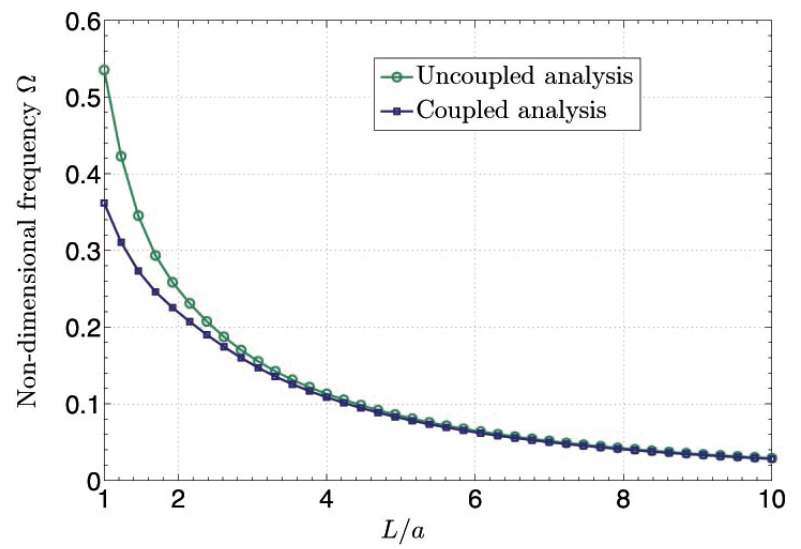

Fig. 17. Frequency curves for flexural modes $(n=1)$, as $\Omega$ versus $L / a$ with $h / a=0.7$.

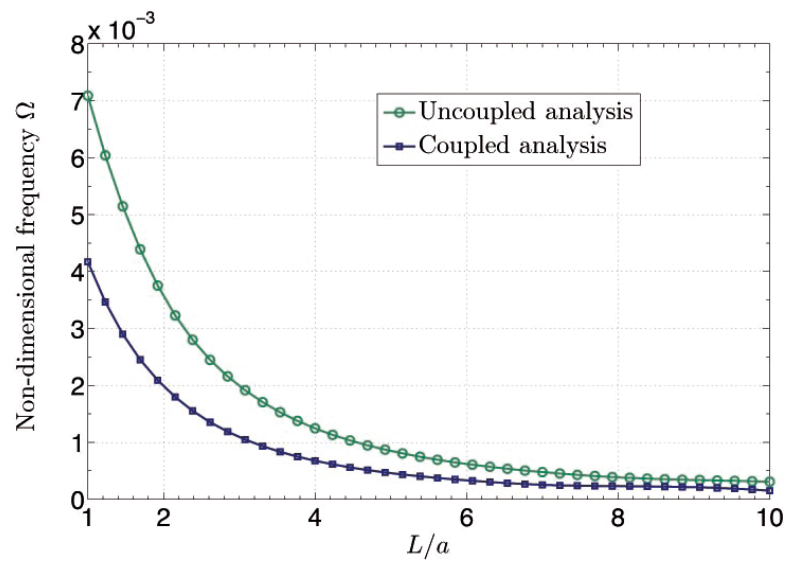

Fig. 18. Frequency curves for lobar modes $(n=2)$, as $\Omega$ versus $L / a$ with $h / a=0.02$.

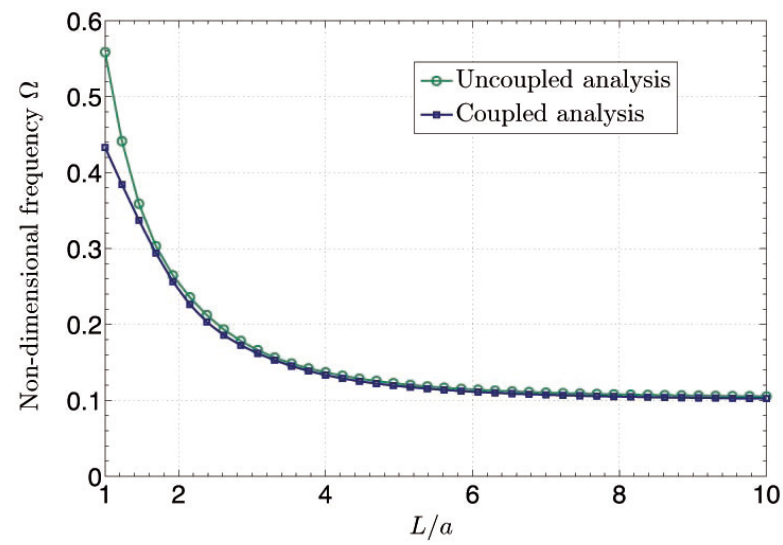

Fig. 19. Frequency curves for lobar modes $(n=2)$, as $\Omega$ versus $L / a$ with $h / a=0.7$. 
For better understanding how the dense fluid (added mass) affects the frequencies, Figs.10 13 have been plotted. Theses figures display the coupled and uncoupled frequencies for $h / a=0.02$ and $h / a=0.7$, with $L / a=1.33$ and $L / a=6$, varying with the circumferential mode $n$. As $n$ increases, the difference between the coupled and uncoupled frequencies reduces at $0<n<3$, but increases after the fundamental frequency at $n=3$ for long thin structures (Fig. 11). For the thick structures, as $n$ increases, the difference between the coupled and uncoupled frequencies reduces at $0<n<6$, but intensifies after the fundamental frequency at $n=6$ for long thick structures (Fig. 13). However, for the short thick structures (Fig. 12), it is observed that as $n$ increases, the difference between the coupled and uncoupled frequencies reduces at $0<n<4$, but amplifies after the fundamental frequency at $n=4$.

A final inspection into cylinder vibrations is given by investigating how the coupled and uncoupled frequencies vary with $L / a$ ratios. Figures 10 12 show the uncoupled and coupled frequencies, with $h / a=0.02$ and $h / a=0.7$, varying with the $L / a$ parameter. The frequency curves of $n=0$ (longitudinal modes), $n=1$ (flexural modes) and $n=2$ (lobar modes) are presented. Due to the coupling effects, all the frequencies decrease as the $L / a$ ratios raise for the curve of $n=0$ (Figs. 14] and 15). It is noted that the frequency spectrum for the vibration modes $n=1$ and $n=2$ for thin structures has similar behavior to that of $n=0$ (Figs. 16] and 18). It can be observed in Figs. 16 19, respectively, that the coupled and uncoupled frequencies, with $h / a=0.02$ and $h / a=0.7$, are varying with the parameter $L / a$ for $n=1$ and $n=2$. For all the above figures, both the coupled and uncoupled frequencies decrease as the $L / a$ ratios increase, but at different rates, the difference between the coupled and uncoupled frequencies reduces as the $L / a$ ratios increase. This shows the different coupling effects on the different mode vibrations $n$ with the $L / a$ ratios. As expected, these figures also register that in the case of thick and long structures, the added mass has no significant effect (Figs. 17 and 19.).

\section{Conclusion}

The characteristics of vibration analysis of two circular cylinders subjected to compressible fluid based on the linear three-dimensional elasticity theory are investigated. The spatial displacement components in the three coordinate directions are described by Helmholtz potential functions. The governing equations are solved to obtain the exact frequency equation. Frequency equations are presented for uncoupled and coupled problems. The nondimensional frequency parameters obtained by the present approach agree very well with those available in the literature in the case of uncoupled problem. However, in the case of coupled problem, the results obtained in this paper can serve as benchmark solutions and as a reference to assess the accuracy of approximate methods. High accuracy have been observed in the analysis. For demonstration, the natural frequencies of different aspect ratios

have been studied in detail, and the first eight frequency parameters for various mode categories are given. The validity of the present solution is solidly confirmed 
numerically. Additionally, the studying of the thermal coupling effect should be explored in the future work.

\section{Acknowledgments}

We would like to sincerely thank Dr. F. Razafimahery for his useful comments, suggestions, agreement and helpful hints to use FEM Comsol Multiphysics.

\section{Appendix A}

A.1. For $i=1,2$, the following formulas may expediently be utilized to obtain the elements of the matrix given in Eq. (17):

$$
\left\{\begin{array}{l}
u_{r}^{(i)}(r) \\
u_{\theta}^{(i)}(r) \\
u_{z}^{(i)}(r) \\
\sigma_{r r}^{(i)}(r) \\
\sigma_{r \theta}^{(i)}(r) \\
\sigma_{r z}^{(i)}(r)
\end{array}\right\}=\left[\begin{array}{cccccc}
u_{1}^{(i)}(r) & u_{2}^{(i)}(r) & u_{3}^{(i)}(r) & u_{4}^{(i)}(r) & u_{5}^{(i)}(r) & u_{6}^{(i)}(r) \\
v_{1}^{(i)}(r) & v_{2}^{(i)}(r) & v_{3}^{(i)}(r) & v_{4}^{(i)}(r) & v_{5}^{(i)}(r) & v_{6}^{(i)}(r) \\
w_{1}^{(i)}(r) & w_{2}^{(i)}(r) & 0 & 0 & w_{5}^{(i)}(r) & w_{6}^{(i)}(r) \\
R_{1}^{(i)}(r) & R_{2}^{(i)}(r) & R_{3}^{(i)}(r) & R_{4}^{(i)}(r) & R_{5}^{(i)}(r) & R_{6}^{(i)}(r) \\
T_{1}^{(i)}(r) & T_{2}^{(i)}(r) & T_{3}^{(i)}(r) & T_{4}^{(i)}(r) & T_{5}^{(i)}(r) & T_{6}^{(i)}(r) \\
Z_{1}^{(i)}(r) & Z_{2}^{(i)}(r) & Z_{3}^{(i)}(r) & Z_{4}^{(i)}(r) & Z_{5}^{(i)}(r) & Z_{6}^{(i)}(r)
\end{array}\right]\left\{\begin{array}{c}
A_{1}^{(i)} \\
A_{2}^{(i)} \\
A_{3}^{(i)} \\
A_{4}^{(i)} \\
A_{5}^{(i)} \\
A_{6}^{(i)}
\end{array}\right\}
$$

A.2. Coefficients of the corresponding displacement components in the radial, circumferential and axial directions (Eq. (13))

$$
\begin{aligned}
u_{1}^{(i)}(r) & =J_{n}^{\prime}\left(k_{\phi_{i}} r\right), & u_{2}^{(i)}(r) & =Y_{n}^{\prime}\left(k_{\phi_{i}} r\right), \\
u_{3}^{(i)}(r) & =-\frac{n}{r} J_{n}\left(k_{\psi_{i}} r\right), & u_{4}^{(i)}(r) & =-\frac{n}{r} Y_{n}\left(k_{\psi_{i}} r\right), \\
u_{5}^{(i)}(r) & =-k_{z} J_{n}^{\prime}\left(k_{\psi_{i}} r\right), & u_{6}^{(i)}(r) & =-k_{z} Y_{n}^{\prime}\left(k_{\psi_{i}} r\right), \\
v_{1}^{(i)}(r) & =\frac{n}{r} J_{n}\left(k_{\phi_{i}} r\right), & v_{2}^{(i)}(r) & =\frac{n}{r} Y_{n}\left(k_{\phi_{i}} r\right), \\
v_{3}^{(i)}(r) & =-J_{n}^{\prime}\left(k_{\psi_{i}} r\right), & v_{4}^{(i)}(r) & =-Y_{n}^{\prime}\left(k_{\psi_{i}} r\right), \\
v_{5}^{(i)}(r) & =-\frac{n k_{z}}{r} J_{n}\left(k_{\psi_{i}} r\right), & v_{6}^{(i)}(r) & =-\frac{n k_{z}}{r} Y_{n}\left(k_{\psi_{i}} r\right), \\
w_{1}^{(i)}(r) & =k_{z} J_{n}\left(k_{\phi_{i}} r\right), & w_{2}^{(i)}(r) & =k_{z} Y_{n}\left(k_{\phi_{i}} r\right), \\
w_{5}^{(i)}(r) & =k_{\psi_{i}}^{2} J_{n}\left(k_{\psi_{i}} r\right), & w_{6}^{(i)}(r) & =k_{\psi_{i}}{ }^{2} Y_{n}\left(k_{\psi_{i}} r\right) .
\end{aligned}
$$


A.3. Coefficients of the pertinent stress components (Eq. (14))

$$
\begin{aligned}
& R_{1}^{(i)}(r)=\mu_{i}\left[2 J_{n}^{\prime \prime}\left(k_{\phi_{i}} r\right)-\frac{\lambda_{i}}{\mu_{i}} \frac{\omega^{2}}{c_{L i}^{2}} J_{n}\left(k_{\phi_{i}} r\right)\right] \\
& R_{2}^{(i)}(r)=\mu_{i}\left[2 Y_{n}^{\prime \prime}\left(k_{\phi_{i}} r\right)-\frac{\lambda_{i}}{\mu_{i}} \frac{\omega^{2}}{c_{L i}^{2}} Y_{n}\left(k_{\phi_{i}} r\right)\right] \\
& R_{3}^{(i)}(r)=-\frac{2 n \mu_{i}}{r^{2}}\left[r J_{n}^{\prime}\left(k_{\psi_{i}} r\right)-J_{n}\left(k_{\psi_{i}} r\right)\right] \\
& R_{4}^{(i)}(r)=-\frac{2 n \mu_{i}}{r^{2}}\left[r Y_{n}^{\prime}\left(k_{\psi_{i}} r\right)-Y_{n}\left(k_{\psi_{i}} r\right)\right] \\
& R_{5}^{(i)}(r)=-2 k_{z} \mu_{i} J_{n}^{\prime \prime}\left(k_{\psi_{i}} r\right) \\
& R_{6}^{(i)}(r)=-2 k_{z} \mu_{i} Y_{n}^{\prime \prime}\left(k_{\psi_{i}} r\right) \\
& T_{1}^{(i)}(r)=\frac{2 n \mu_{i}}{r^{2}}\left[r J_{n}^{\prime}\left(k_{\phi_{i}} r\right)-J_{n}\left(k_{\phi_{i}} r\right)\right] \\
& T_{2}^{(i)}(r)=\frac{2 n \mu_{i}}{r^{2}}\left[r Y_{n}^{\prime}\left(k_{\phi_{i}} r\right)-Y_{n}\left(k_{\phi_{i}} r\right)\right] \\
& T_{3}^{(i)}(r)=\mu_{i}\left[-2 J_{n}^{\prime \prime}\left(k_{\psi_{i}} r\right)-k_{\psi_{i}}^{2} J_{n}\left(k_{\psi_{i}} r\right)\right] \\
& T_{4}^{(i)}(r)=\mu_{i}\left[-2 Y_{n}^{\prime \prime}\left(k_{\psi_{i}} r\right)-k_{\psi_{i}}^{2} Y_{n}\left(k_{\psi_{i}} r\right)\right] \\
& T_{5}^{(i)}(r)=-\frac{2 n \mu_{i} k_{z}}{r^{2}}\left[r J_{n}^{\prime}\left(k_{\psi_{i}} r\right)-J_{n}\left(k_{\psi_{i}} r\right)\right] \\
& T_{6}^{(i)}(r)=-\frac{2 n \mu_{i} k_{z}}{r^{2}}\left[r Y_{n}^{\prime}\left(k_{\psi_{i}} r\right)-Y_{n}\left(k_{\psi_{i}} r\right)\right] \\
& Z_{1}^{(i)}(r)=2 k_{z} \mu_{i} J_{n}^{\prime}\left(k_{\phi_{i}} r\right) \\
& Z_{2}^{(i)}(r)=2 k_{z} \mu_{i} Y_{n}^{\prime}\left(k_{\phi_{i}} r\right) \\
& Z_{3}^{(i)}(r)=-\frac{n \mu_{i} k_{z}}{r} J_{n}\left(k_{\psi_{i}} r\right) \\
& Z_{4}^{(i)}(r)=-\frac{n \mu_{i} k_{z}}{r} Y_{n}\left(k_{\psi_{i}} r\right) \\
& Z_{5}^{(i)}(r)=\mu_{i}\left(k_{\psi_{i}}^{2}-k_{z}^{2}\right) J_{n}^{\prime}\left(k_{\psi_{i}} r\right) \\
& Z_{6}^{(i)}(r)=\mu_{i}\left(k_{\psi_{i}}^{2}-k_{z}^{2}\right) Y_{n}^{\prime}\left(k_{\psi_{i}} r\right)
\end{aligned}
$$




\section{A.4. Elements of the matrix given in Eq. (17)}

$$
\begin{aligned}
& N_{11}^{(i)}=R_{1}^{(i)}(a), \quad N_{12}^{(i)}=R_{2}^{(i)}(a), \quad N_{13}^{(i)}=R_{3}^{(i)}(a), \quad N_{14}^{(i)}=R_{4}^{(i)}(a) \\
& N_{15}^{(i)}=R_{5}^{(i)}(a), \quad N_{16}^{(i)}=R_{6}^{(i)}(a), \quad N_{1}=J_{n}\left(k_{\Phi} r\right) \\
& N_{21}^{(i)}=T_{1}^{(i)}(a), \quad N_{22}^{(i)}=T_{2}^{(i)}(a), \quad N_{23}^{(i)}=T_{3}^{(i)}(a), \quad N_{24}^{(i)}=T_{4}^{(i)}(a) \\
& N_{25}^{(i)}=T_{5}^{(i)}(a), \quad N_{26}^{(i)}=T_{6}^{(i)}(a) \\
& N_{31}^{(i)}=Z_{1}^{(i)}(a), \quad N_{32}^{(i)}=Z_{2}^{(i)}(a), \quad N_{33}^{(i)}=Z_{3}^{(i)}(a), \quad N_{34}^{(i)}=Z_{4}^{(i)}(a) \\
& N_{35}^{(i)}=Z_{5}^{(i)}(a), \quad N_{36}^{(i)}=Z_{6}^{(i)}(a) \\
& N_{41}^{(i)}=u_{1}^{(i)}(a), \quad N_{42}^{(i)}=u_{2}^{(i)}(a), \quad N_{43}^{(i)}=u_{3}^{(i)}(a), \quad N_{44}^{(i)}=u_{4}^{(i)}(a) \\
& N_{45}^{(i)}=u_{5}^{(i)}(a), \quad N_{46}^{(i)}=u_{6}^{(i)}(a), \quad N_{4}=-\frac{J_{n}^{\prime}\left(k_{\Phi} r\right)}{\rho_{0} \omega^{2}} \\
& N_{51}^{(i)}=R_{1}^{(i)}\left(a_{1}\right), \quad N_{52}^{(i)}=R_{2}^{(i)}\left(a_{1}\right), \quad N_{53}^{(i)}=R_{3}^{(i)}\left(a_{1}\right), \quad N_{54}^{(i)}=R_{4}^{(i)}\left(a_{1}\right) \\
& N_{55}^{(i)}=R_{5}^{(i)}\left(a_{1}\right), \quad N_{56}^{(i)}=R_{6}^{(i)}\left(a_{1}\right) \\
& N_{61}^{(i)}=T_{1}^{(i)}\left(a_{1}\right), \quad N_{62}^{(i)}=T_{2}^{(i)}\left(a_{1}\right), \quad N_{63}^{(i)}=T_{3}^{(i)}\left(a_{1}\right), \quad N_{64}^{(i)}=T_{4}^{(i)}\left(a_{1}\right) \\
& N_{65}^{(i)}=T_{5}^{(i)}\left(a_{1}\right), \quad N_{66}^{(i)}=T_{6}^{(i)}\left(a_{1}\right) \\
& N_{71}^{(i)}=Z_{1}^{(i)}\left(a_{1}\right), \quad N_{72}^{(i)}=Z_{2}^{(i)}\left(a_{1}\right), \quad N_{73}^{(i)}=Z_{3}^{(i)}\left(a_{1}\right), \quad N_{74}^{(i)}=Z_{4}^{(i)}\left(a_{1}\right) \\
& N_{75}^{(i)}=Z_{5}^{(i)}\left(a_{1}\right), \quad N_{76}^{(i)}=Z_{6}^{(i)}\left(a_{1}\right) \\
& N_{81}^{(i)}=u_{1}^{(i)}\left(a_{1}\right), \quad N_{82}^{(i)}=u_{2}^{(i)}\left(a_{1}\right), \quad N_{83}^{(i)}=u_{3}^{(i)}\left(a_{1}\right), \quad N_{84}^{(i)}=u_{4}^{(i)}\left(a_{1}\right) \\
& N_{85}^{(i)}=u_{5}^{(i)}\left(a_{1}\right), \quad N_{86}^{(i)}=u_{6}^{(i)}\left(a_{1}\right) \\
& N_{91}^{(i)}=v_{1}^{(i)}\left(a_{1}\right), \quad N_{92}^{(i)}=v_{2}^{(i)}\left(a_{1}\right), \quad N_{93}^{(i)}=v_{3}^{(i)}\left(a_{1}\right), \quad N_{94}^{(i)}=v_{4}^{(i)}\left(a_{1}\right) \\
& N_{95}^{(i)}=v_{5}^{(i)}\left(a_{1}\right), \quad N_{96}^{(i)}=v_{6}^{(i)}\left(a_{1}\right) \\
& N_{101}^{(i)}=w_{1}^{(i)}\left(a_{1}\right), \quad N_{102}^{(i)}=w_{2}^{(i)}\left(a_{1}\right), \quad N_{105}^{(i)}=w_{5}^{(i)}\left(a_{1}\right), \quad N_{106}^{(i)}=w_{6}^{(i)}\left(a_{1}\right) \\
& N_{111}^{(i)}=R_{1}^{(i)}\left(a_{2}\right), \quad N_{112}^{(i)}=R_{2}^{(i)}\left(a_{2}\right), \quad N_{113}^{(i)}=R_{3}^{(i)}\left(a_{2}\right), \quad N_{114}^{(i)}=R_{4}^{(i)}\left(a_{2}\right) \\
& N_{115}^{(i)}=R_{5}^{(i)}\left(a_{2}\right), \quad N_{116}^{(i)}=R_{6}^{(i)}\left(a_{2}\right) \\
& N_{121}^{(i)}=T_{1}^{(i)}\left(a_{2}\right), \quad N_{122}^{(i)}=T_{2}^{(i)}\left(a_{2}\right), \quad N_{123}^{(i)}=T_{3}^{(i)}\left(a_{2}\right), \quad N_{124}^{(i)}=T_{4}^{(i)}\left(a_{2}\right) \\
& N_{125}^{(i)}=T_{5}^{(i)}\left(a_{2}\right), \quad N_{126}^{(i)}=T_{6}^{(i)}\left(a_{2}\right) \\
& N_{131}^{(i)}=Z_{1}^{(i)}\left(a_{2}\right), \quad N_{132}^{(i)}=Z_{2}^{(i)}\left(a_{2}\right), \quad N_{133}^{(i)}=Z_{3}^{(i)}\left(a_{2}\right), \quad N_{134}^{(i)}=Z_{4}^{(i)}\left(a_{2}\right) \\
& N_{135}^{(i)}=Z_{5}^{(i)}\left(a_{2}\right), \quad N_{136}^{(i)}=Z_{6}^{(i)}\left(a_{2}\right)
\end{aligned}
$$




\section{References}

Alibeigloo, A. and Jafarian, H. [2016] "Three-dimensional static and free vibration analysis of carbon nano tube reinforced composite cylindrical shell using differential quadrature method," International Journal of Applied Mechanics 08, 1650033.

Armenakas, A. E. [1967] "Propagation of harmonic waves in composite circular cylindrical shells. Part I: Theoretical investigation," AIAA Journal 5(4), 740-744.

Armenakas, A. E., Gazis, D. C. and Herrmann, G. [1969] Free Vibrations of Circular Cylindrical Shells (Pergamon Press, Oxford).

Brischetto, S. [2014] "An exact 3D solution for free vibrations of multilayered cross-ply composite and sandwich plates and shells," International Journal of Applied Mechanics 06, 1450076.

Comsol Multiphysics User's Guide [2016] Version 5.2 (Stockholm, Sweden).

El Baroudi, A., Razafimahery, F. and Rakotomanana, L. [2014] "Fluid structure interaction within three-dimensional models of an idealized arterial wall," International Journal of Engineering Science 84, 113-126.

Gazis, D. C. [1959a] "Three-dimensional investigation of the propagation of waves in hollow circular cylinders, I: Analytical foundation," Journal of the Acoustical Society of America 31(5), 568-573.

Gazis, D. C. [1959b] "Three-dimensional investigation of the propagation of waves in hollow circular cylinders, II: Numerical results," Journal of the Acoustical Society of America 31(5), 573-578.

Gladwell, G. M. L. and Vijay, D. K. [1975] "Natural frequencies of free finite length circular cylinders," Journal of Sound and Vibration 42(3), 387-397.

Hamidzadeh, H. R. and Jazar, R. N. [2010] Vibrations of Thick Cylindrical Structures (Springer, New York).

Leissa, A. W. [1973] Vibration of Shells (NASA SP-288, US Government Printing Office).

McNiven, H. D. and Perry, D. C. [1962] "Axially symmetric waves in finite, elastic rods," Journal of the Acoustical Society of America 34, 433-437.

McNiven, H. D., Shah, A. H. and Sackman, J. L. [1966] "Axially symmetric waves in hollow, elastic rods: Part I," Journal of the Acoustical Society of America 40, 784-791.

Morse, M. and Feshbach, H. R. [1946] Methods of Theoretical Physics (McGraw-Hill, New York).

Oliazadeh, P., Farshidianfar, M. H. and Farshidianfar, A. [2013] "Exact analysis of resonance frequency and mode shapes of isotropic and laminated composite cylindrical shells; Part I: Analytical studies," Journal of Mechanical Science and Technology 27(12), 3635-3643.

Pierce, A. D. [1991] Acoustics: An Introduction to Its Physical Principles and Applications (American Institute of Physics, New York).

Qu, Y., Chen, Y., Long, X., Hua, H. and Meng, G. [2013] "Free and forced vibration analysis of uniform and stepped circular cylindrical shells using a domain decomposition method," Applied Acoustics 74, 425-439.

Singal, R. K. and Williams, K. [1988] "A theoretical and experimental study of vibrations of thick circular cylindrical shells and rings," Journal of Vibration, Acoustics, Stress and Reliability 110(4), 533-537.

Wolfram Research Mathematica [2015] User's Manual Version 10.1, Champaign, Illinois.

Xuebin, L. [2008] "Study on free vibration analysis of circular cylindrical shells using wave propagation," Journal of Sound and Vibration 311, 667-682. 
Ye, T., Jin, G., Shi, S. and Xianglong, M. [2014] "Three-dimensional free vibration analysis of thick cylindrical shells with general end conditions and resting on elastic foundations," International Journal of Mechanical Sciences 84, 120-137.

Zhang, X. M. [2002] "Frequency analysis of submerged cylindrical shells with the wave propagation approach," International Journal of Mechanical Sciences 44, 1259-1273. 